\title{
ANISOTROPIC ESTIMATES FOR THE TWO-DIMENSIONAL KURAMOTO-SIVASHINSKY EQUATION
}

\author{
SAID BENACHOUR, IGOR KUKAVICA, WALTER RUSIN, AND MOHAMMED ZIANE
}

\begin{abstract}
We address the global solvability of the Kuramoto-Sivashinsky equation in a rectangular domain $\left[0, L_{1}\right] \times\left[0, L_{2}\right]$. We give sufficient conditions on the width $L_{2}$ of the domain, depending on the length $L_{1}$, so that the obtained solutions are global. Our proofs are based on anisotropic estimates.
\end{abstract}

\section{INTRODUCTION}

In this paper, we consider the Kuramoto-Sivashinsky equation in a two-dimensional domain $\Omega=\left[0, L_{1}\right] \times\left[0, L_{2}\right]$

$$
\partial_{t} \varphi+\Delta^{2} \varphi+\Delta \varphi+\frac{1}{2}|\nabla \varphi|^{2}=0
$$

with periodic boundary conditions. The equation is supplemented with the initial condition

$$
\varphi(x, y, 0)=\varphi_{0}(x, y) .
$$

Due to the symmetry of the equation (KS) with respect to the spatial variables we may assume, without loss of generality, that $L_{2} \leq L_{1}$. We shall study the equation satisfied by $u=\left(u_{1}, u_{2}\right)=$ $\nabla \varphi$. In the two-dimensional case, it reads

$$
\begin{aligned}
& \partial_{t} u_{1}+\Delta^{2} u_{1}+\Delta u_{1}+u_{1} \partial_{x} u_{1}+u_{2} \partial_{x} u_{2}=0 \\
& \partial_{t} u_{2}+\Delta^{2} u_{2}+\Delta u_{2}+u_{1} \partial_{y} u_{1}+u_{2} \partial_{y} u_{2}=0 \\
& \partial_{y} u_{1}=\partial_{x} u_{2}
\end{aligned}
$$

The role of the Kuramoto-Sivashinsky equation in the contemporary nonlinear mechanics and physics is well known. It arises as a model in hydrodynamics (a thin film flow down an inclined plane in the presence of an electric field), in combustion theory (propagation of flame fronts), phase turbulence and plasma physics, as well as a model for spatio-temporal chaos; c.f. $[2,14]$ for a short review of applications with key references.

The Kuramoto-Sivashinsky equation has been extensively studied in dimension one. In the middle eighties, Nicolaenko, Scheurer, and Temam in [12] showed, that when the dimension is less than or equal to 3, the existence of a global absorbing ball implies the existence of a global attractor and they provided an upper estimate on its Hausdorff dimension. They also proved that, under the

Date: October 18, 2012. 
assumption that the initial value for $u$ is odd, the existence of a bounded global absorbing set in $L_{\text {per }}^{2}([0, L])$ for the equation satisfied by $u=\varphi_{x}$ in dimension 1 . This antisymmetry requirement was later removed by Collet, Eckmann, Epstein, and Stubbe [3] and, independently, by Goodman [5] (c.f. [8]). The strategy in $[3,5,12]$ is based on an ingenious Lyapunov-type argument. More recently, Giacomelli and Otto [4] improved these results by treating the Kuramoto-Sivashinsky equation as a perturbation of the inviscid Burgers equation; c.f. also a recent paper by Otto [13].

Since the Kuramoto-Sivashinsky equation models the flame propagation fronts, the main physical interest is in the two dimensional case. However, the global well-posedness for (1.2) in 2D is an open problem. This is related to the fact that, although the equation is locally well-posed in $L^{2}(\Omega)$, it does not preserve the $L^{2}$-norm (see Section 2 for more details). A first global well-posedness result was given by Sell and Taboada in [19], where they showed the existence of a bounded local absorbing set in $H_{\text {per }}^{1}([0,2 \pi] \times[0,2 \pi \epsilon])$ for $\epsilon$ small enough by adapting the method developed by Raugel and Sell [16] for the Navier-Stokes equations in a thin domain of $\mathbb{R}^{3}$ (c.f. also $[1,6,7,15,17]$ ). In [11] Molinet obtained a more transparent result on the local dissipativity of the (KS) equation in a thin rectangular domain and gave a sufficient condition on $L_{2}$, depending on $L_{1}$, so that the equation (1.2) admits a global solution. Assuming that $L_{2}<C^{-1} L_{1}^{-67 / 35}$, the solutions are global provided that $\left\|u_{1}(0)\right\|_{L^{2}(\Omega)}$ is of order $C^{-1} L_{1}^{-1 / 4} L_{2}^{-7 / 4}$, whereas $\left\|u_{2}(0)\right\|_{L^{2}(\Omega)}$ is of order $C^{-1} L_{1}^{-1 / 4} L_{2}^{-1 / 4}$.

In this paper, we prove the global existence for data in the domain $\left[0, L_{1}\right] \times\left[0, L_{2}\right]$ when $L_{2} \leq$

$C^{-1} L_{1}^{-22 / 25}$ where $L_{1} \geq 2 \pi$ is arbitrary, and where the $L^{2}$ norm of the initial data is bounded by $C^{-1} L_{2}^{-2}$. This allows larger initial data than previously known.

The rest of the paper is organized as follows. In Section 2 we introduce the notation, functional spaces, and define the notion of a solution. Section 3 includes the statement of the main result. In Section 4 we recall some auxiliary results used in the proof of the main result. Finally, Section 5 is devoted to the proof of the main theorem.

\section{Notation}

For $m \in \mathbb{N}$, we denote by $H_{\text {per }}^{m}(\Omega)$ the Sobolev space of $\Omega$-periodic distributions whose derivatives up to order $m$ belong to $L_{\text {per }}^{2}(\Omega)$.

Definition 2.1. A function $u(t, x, y)$ is a weak solution of (1.2) if $u \in C\left([0, T], L_{\text {per }}^{2}(\Omega)\right) \cap$ $L^{2}\left((0, T), H_{\mathrm{per}}^{2}(\Omega)\right)$ and (1.2) is satisfied in the sense of distributions in $\left(0, L_{1}\right) \times\left(0, L_{2}\right) \times(0, T)$.

For any $f \in L_{\text {per }}^{1}(\Omega)$, define

$$
M[f](x)=\frac{1}{L_{2}} \int_{0}^{L_{2}} f(x, \tau) d \tau
$$


and

$$
N[f](x, y)=f(x, y)-M[f](x),
$$

which exist for almost all $(x, y) \in\left[0, L_{1}\right] \times\left[0, L_{2}\right]$. Similarly, we write the average in the horizontal direction as

$$
M_{x}[f](y)=\frac{1}{L_{1}} \int_{0}^{L_{1}} f(\tau, y) d \tau
$$

and

$$
N_{x}[f](x, y)=f(x, y)-M_{x}[f](y) .
$$

\section{MAin RESUlts}

The following is the main result of the paper.

Theorem 3.1. There exists a positive constants $C_{0} \geq 1$ such that, for any $L_{1} \geq 2 \pi$, if

$$
L_{2} \leq C_{0}^{-1} L_{1}^{-22 / 25}
$$

then the solution of (1.2) associated with the initial datum $u_{0}=\left(u_{01}, u_{02}\right) \in L^{2}(\Omega)$ with $\int_{\Omega} u_{0}=0$, curl $u_{0}=0$, and

$$
\begin{aligned}
\left\|u_{01}\right\|_{L^{2}(\Omega)} & \leq C_{0}^{-1} L_{2}^{-2}, \\
\left\|u_{02}\right\|_{L^{2}(\Omega)} & \leq C_{0}^{-1} L_{2}^{-2}
\end{aligned}
$$

is global in time.

\section{Preliminaries}

Before we prove the theorem, we recall a few useful auxiliary results.

Proposition 4.1. Let $u_{0}(\cdot)=\left(u_{01}(\cdot), u_{02}(\cdot)\right) \in\left(L_{\text {per }}^{2}(\Omega)\right)^{2}$ such that curl $u_{0}=0$. There exists $T=T\left(\left\|u_{0}\right\|_{L^{2}(\Omega)}\right)$ such that the Cauchy problem (1.2) with the initial condition $u(0, \cdot)=u_{0}(\cdot)$ possesses a unique weak solution on $[0, T] \times \Omega$.

Proof. Proof of the proposition can be found for instance in [2].

Remark 4.2. By the embedding theorem and classical parabolic smoothing properties, this local solution $u(t)$ belongs to $H_{\mathrm{per}}^{4}(\Omega)$ for $t>0$. This regularity permits the calculations below.

Lemma 4.3. We have

$$
\|N[f]\|_{L^{2}(\Omega)} \leq C L_{2}\left\|\partial_{y} N[f]\right\|_{L^{2}(\Omega)},
$$

for $f \in H_{\mathrm{per}}^{1}(\Omega)$. 
Proof. Proof of the lemma can be found in [22].

Regarding the linear problem associated with the one-dimensional equation (KS) written in the frequency space

$$
\partial_{t} \widehat{\varphi}=\left(k^{2}-k^{4}\right) \widehat{\varphi}
$$

one observes that the linear operator amplifies the low frequencies but damps the high ones. The unstable frequencies are $O(1)$ and have a growth rate of $O(1)$. Therefore the $L^{2}$-norm of the solutions to the one-dimensional Kuramoto-Sivashinsky may in principle grow exponentially. This effect is present in the energy estimate of $M\left[u_{1}\right]$, which is the two-dimensional part of $u_{1}$. In order to obtain the necessary estimates, we employ techniques developed by Nicolaenko, Scheurer, and Temam in [12] and Goodman in [5].

For a smooth $L_{1}$-periodic function $\Phi$ depending only on $x$, which will be specified in Proposition 4.4, we denote

$$
\mathcal{T}=\left\{\Phi(x+b), b \in\left[0, L_{1}\right]\right\}
$$

Also, let

$$
\begin{gathered}
F\left(u_{1}\right)(t)=\inf _{b \in\left[0, L_{1}\right]} \Gamma(b)=\inf _{b \in\left[0, L_{1}\right]} \int_{\Omega}\left|M\left[u_{1}\right](x, t)-\Phi(x+b)\right|^{2} d x d y \\
=\int_{\Omega}\left|M\left[u_{1}\right](x, t)-\Phi\left(x+b^{*}\right)\right|^{2} d x d y
\end{gathered}
$$

be the square of the distance in $L^{2}(\Omega)$ between $M\left[u_{1}\right]$ and $\mathcal{T}$. Since the function $b \mapsto \Gamma(b)$ belong to $C_{\mathrm{per}}^{1}\left(\left[0, L_{1}\right]\right)$ there exists at least one optimal translation $b^{*}$, and any optimal translation $b^{*}$ satisfies

$$
\int_{\Omega} M\left[u_{1}\right](x, t) \partial_{x} \Phi\left(x+b^{*}\right) d x d y=\int_{\Omega}\left(M\left[u_{1}\right](x, t)-\Phi\left(x+b^{*}\right)\right) \partial_{x} \Phi\left(x+b^{*}\right) d x d y=0
$$

(c.f. [5]). Naturally $b^{*}=b^{*}(t)$ may change in time. However for any time $t_{0}>0$ in the maximal interval of existence of $u$, denoting $\Phi_{b^{*}\left(t_{0}\right)}(x)=\Phi\left(x+b^{*}\left(t_{0}\right)\right)$, we have

$$
\begin{gathered}
\left.\frac{d}{d t} F\left(u_{1}\right)(\cdot)\right|_{t=t_{0}} \leq\left.\frac{d}{d t}\left\|M\left[u_{1}\right](\cdot)-\Phi_{b^{*}\left(t_{0}\right)}\right\|_{L^{2}(\Omega)}^{2}\right|_{t=t_{0}} \\
=\left.2 \int_{\Omega} \partial_{t} M\left[u_{1}\right](\cdot)\right|_{t=t_{0}}\left(M\left[u_{1}\right]\left(t_{0}\right)-\Phi_{b^{*}\left(t_{0}\right)}\right) .
\end{gathered}
$$

The left side of (4.14) should be understood as the upper right derivative $\frac{d}{d t_{+}}$. For more details, we refer the interested reader to [9]. 
For a smooth function $\Phi$, introduce the bilinear form

$$
\begin{aligned}
(f, g)_{\Phi}=\int_{\Omega} \partial_{x x} f \partial_{x x} g-\int_{\Omega} \partial_{x} f \partial_{x} g+\int_{\Omega} f g \partial_{x} \Phi & \\
& +\frac{4}{L_{1}} \int_{0}^{L_{2}}\left(\left(\int_{0}^{L_{1}} f \partial_{x} \Phi d x\right)\left(\int_{0}^{L_{1}} g \partial_{x} \Phi d x\right)\right) d y
\end{aligned}
$$

and the associated quadratic form

$$
\mathcal{B}_{\Phi}(f)=(f, f)_{\Phi}
$$

First, we recall the proposition addressing the positivity of the bilinear form.

Proposition 4.4. There exists a constant $C \geq 1$ such that the following property holds: For any pair $\left(L_{1}, L_{2}\right) \in \mathbb{R}_{+} \times \mathbb{R}_{+}$there exists a smooth function $\Phi: \Omega \rightarrow \mathbb{R}$ depending only on $x$, with the

property $\int_{0}^{L_{1}} \Phi(x) d x=0$, such that for any $\gamma \in[1 / 4,1]$ and any $f \in H^{2}(\Omega)$ depending only on $x$, we have

$$
\mathcal{B}_{\gamma \Phi}(f) \geq \frac{1}{3}\left\|\partial_{x x} f\right\|_{L^{2}(\Omega)}^{2}+3\|f\|_{L^{2}(\Omega)}^{2}
$$

with

$$
\|\Phi\|_{L^{2}(\Omega)}^{2} \leq C L_{1}^{8 / 5} L_{2}
$$

and

$$
\left\|\partial_{x x} \Phi\right\|^{2} \leq C L_{1}^{16 / 5} L_{2}
$$

Proof. The proof of the proposition can be found in [3].

\section{Proof of main Result}

In this section, we prove the main result of this paper. Throughout the following, $C$ denotes a positive constant.

Proof of Theorem 3.1. First, we note that equation (1.2) 3 yields

$$
\partial_{x} M\left[u_{2}\right]=\partial_{y} M\left[u_{1}\right]=0 .
$$

Therefore, using $\int_{\Omega} u=0$, we obtain

$$
u_{2}=N\left[u_{2}\right]
$$

Similarly, we have

$$
u_{1}=N_{x}\left[u_{1}\right]
$$


We multiply the equation $(1.2)_{2}$ by $u_{2}$ and integrate by parts in $\Omega$. We get

$$
\frac{1}{2} \frac{d}{d t}\left\|u_{2}\right\|_{L^{2}(\Omega)}^{2}+\left\|\Delta u_{2}\right\|_{L^{2}(\Omega)}^{2}-\left\|\nabla u_{2}\right\|_{L^{2}(\Omega)}^{2}=-\int_{\Omega} u_{1} \partial_{y} u_{1} u_{2} .
$$

In order to estimate the integral on the right side of (5.23), we note that

$$
-\int_{\Omega} u_{1} \partial_{y} u_{1} u_{2}=-\int_{\Omega} u_{1} \partial_{y} u_{1} N\left[u_{2}\right]=-\int_{\Omega} u_{1} \partial_{y} N\left[u_{1}\right] N\left[u_{2}\right]=-\int_{\Omega} u_{1} \partial_{x} N\left[u_{2}\right] N\left[u_{2}\right],
$$

where we used (5.21). By Hölder's inequality

$$
\begin{aligned}
& -\int_{\Omega} u_{1} \partial_{x} N\left[u_{2}\right] N\left[u_{2}\right] \leq \int_{0}^{L_{1}}\left\|u_{1}\right\|_{L_{y}^{2}\left(\left[0, L_{2}\right]\right)}\left\|\partial_{x} N\left[u_{2}\right]\right\|_{L_{y}^{2}\left(\left[0, L_{2}\right]\right)}\left\|N\left[u_{2}\right]\right\|_{L_{y}^{\infty}\left(\left[0, L_{2}\right]\right)} d x \\
& \quad \leq C \int_{0}^{L_{1}}\left\|u_{1}\right\|_{L_{y}^{2}\left(\left[0, L_{2}\right]\right)}\left\|\partial_{x} N\left[u_{2}\right]\right\|_{L_{y}^{2}\left(\left[0, L_{2}\right]\right)}\left\|N\left[u_{2}\right]\right\|_{L_{y}^{2}\left(\left[0, L_{2}\right]\right)}^{1 / 2}\left\|\partial_{y} N\left[u_{2}\right]\right\|_{L_{y}^{2}\left(\left[0, L_{2}\right]\right)}^{1 / 2} d x \\
& \quad \leq C\left\|u_{1}\right\|_{L^{2}(\Omega)}\left\|\partial_{x} N\left[u_{2}\right]\right\|_{L_{y}^{2} L_{x}^{\infty}(\Omega)}\left\|N\left[u_{2}\right]\right\|_{L^{2}(\Omega)}^{1 / 2}\left\|\partial_{y} N\left[u_{2}\right]\right\|_{L^{2}(\Omega)}^{1 / 2},
\end{aligned}
$$

where we used Agmon's inequality in the $y$-variable and Hölder's inequality. By Agmon's inequality in the $x$-variable, we may bound the far right side of (5.25) by

$$
C\left\|u_{1}\right\|_{L^{2}(\Omega)}\left\|\partial_{x} N\left[u_{2}\right]\right\|_{L^{2}(\Omega)}^{1 / 2}\left\|\partial_{x x} N\left[u_{2}\right]\right\|_{L^{2}(\Omega)}^{1 / 2}\left\|N\left[u_{2}\right]\right\|_{L^{2}(\Omega)}^{1 / 2}\left\|\partial_{y} N\left[u_{2}\right]\right\|_{L^{2}(\Omega)}^{1 / 2},
$$

where we used $\partial_{x} M_{x}[\cdot] \equiv 0$. Using Lemma 4.3 we estimate the above expression by

$$
\begin{aligned}
& C\left\|u_{1}\right\|_{L^{2}(\Omega)} L_{2}^{1 / 2}\left\|\partial_{y x} N\left[u_{2}\right]\right\|_{L^{2}(\Omega)}^{1 / 2}\left\|\partial_{x x} N\left[u_{2}\right]\right\|_{L^{2}(\Omega)}^{1 / 2} L_{2}\left\|\partial_{y y} N\left[u_{2}\right]\right\|_{L^{2}(\Omega)}^{1 / 2} L_{2}^{1 / 2}\left\|\partial_{y y} N\left[u_{2}\right]\right\|_{L^{2}(\Omega)}^{1 / 2} \\
& \quad \leq C L_{2}^{2}\left\|u_{1}\right\|_{L^{2}(\Omega)}\left\|\Delta u_{2}\right\|_{L^{2}(\Omega)}^{2} .
\end{aligned}
$$

Thus, by (5.24)-(5.27) and the triangle inequality

$$
\begin{gathered}
-\int_{\Omega} u_{1} \partial_{y} u_{1} u_{2} \leq C L_{2}^{2}\left(\left\|N\left[u_{1}\right]\right\|_{L^{2}(\Omega)}+\left\|M\left[u_{1}\right]\right\|_{L^{2}(\Omega)}\right)\left\|\Delta u_{2}\right\|_{L^{2}(\Omega)}^{2} \\
\leq C L_{2}^{2}\left(\|\Phi\|_{L^{2}(\Omega)}+\left\|N\left[u_{1}\right]\right\|_{L^{2}(\Omega)}+\sqrt{F\left(u_{1}\right)}\right)\left\|\Delta u_{2}\right\|_{L^{2}(\Omega)}^{2} .
\end{gathered}
$$

Combining (5.23) and (5.28), we obtain

$$
\begin{aligned}
& \frac{1}{2} \frac{d}{d t}\left\|u_{2}\right\|_{L^{2}(\Omega)}^{2}+\left\|\Delta u_{2}\right\|_{L^{2}(\Omega)}^{2}-\left\|\nabla u_{2}\right\|_{L^{2}(\Omega)}^{2} \\
& \quad \leq C L_{2}^{2}\left(\|\Phi\|_{L^{2}(\Omega)}+\left\|N\left[u_{1}\right]\right\|_{L^{2}(\Omega)}+\sqrt{F\left(u_{1}\right)}\right)\left\|\Delta u_{2}\right\|_{L^{2}(\Omega)}^{2} .
\end{aligned}
$$

Due to (5.21) we may use the Poincaré inequality $\left\|\nabla u_{2}\right\|_{L^{2}(\Omega)}^{2} \leq\left(L_{2} / 2 \pi\right)^{2}\left\|\Delta u_{2}\right\|_{L^{2}(\Omega)}^{2}$ and by the assumption $L_{2}<1$, it follows that

$$
\begin{aligned}
& \frac{1}{2} \frac{d}{d t}\left\|u_{2}\right\|_{L^{2}(\Omega)}^{2}+\frac{1}{2}\left\|\Delta u_{2}\right\|_{L^{2}(\Omega)}^{2} \\
& \quad \leq-\left(\frac{1}{2}-\left(\frac{L_{2}}{2 \pi}\right)^{2}-C L_{2}^{2}\left(\|\Phi\|_{L^{2}(\Omega)}+\left\|N\left[u_{1}\right]\right\|_{L^{2}(\Omega)}+\sqrt{F\left(u_{1}\right)}\right)\right)\left\|\Delta u_{2}\right\|_{L^{2}(\Omega)}^{2} .
\end{aligned}
$$


In order to obtain an estimate of $N\left[u_{1}\right]$, we apply the operator $N[\cdot]$ to $(1.2)_{1}$, multiply the resulting equation by $N\left[u_{1}\right]$, and integrate over $\Omega$. We get

$$
\begin{gathered}
\frac{1}{2} \frac{d}{d t}\left\|N\left[u_{1}\right]\right\|_{L^{2}(\Omega)}^{2}+\left\|\Delta N\left[u_{1}\right]\right\|_{L^{2}(\Omega)}^{2}-\left\|\nabla N\left[u_{1}\right]\right\|_{L^{2}(\Omega)}^{2} \\
=-\int_{\Omega} N\left[u_{1} \partial_{x} u_{1}\right] N\left[u_{1}\right]-\int_{\Omega} N\left[u_{2} \partial_{x} u_{2}\right] N\left[u_{1}\right] .
\end{gathered}
$$

Regarding the first term on the right side of (5.31), we have

$$
\begin{aligned}
-\int_{\Omega} N\left[u_{1} \partial_{x} u_{1}\right] N\left[u_{1}\right]= & -\int_{\Omega} N\left[N\left[u_{1}\right] \partial_{x} N\left[u_{1}\right]\right] N\left[u_{1}\right]-\int_{\Omega} N\left[M\left[u_{1}\right] \partial_{x} M\left[u_{1}\right]\right] N\left[u_{1}\right] \\
& -\int_{\Omega} N\left[M\left[u_{1}\right] \partial_{x} N\left[u_{1}\right]\right] N\left[u_{1}\right]-\int_{\Omega} N\left[N\left[u_{1}\right] \partial_{x} M\left[u_{1}\right]\right] N\left[u_{1}\right] \\
& =I+I I+I I I+I V .
\end{aligned}
$$

First, we note that for the term $I$, we obtain

$$
I=-\int_{\Omega} N\left[N\left[u_{1}\right] \partial_{x} N\left[u_{1}\right]\right] N\left[u_{1}\right]=-\int_{\Omega} N\left[u_{1}\right] \partial_{x} N\left[u_{1}\right] N\left[u_{1}\right]=0,
$$

where we used $N[N[\cdot]]=N[\cdot]$. Next, regarding the term $I I$ we observe that

$$
-\int_{\Omega} N\left[M\left[u_{1}\right] \partial_{x} M\left[u_{1}\right]\right] N\left[u_{1}\right]=0 .
$$

In order to obtain an estimate of the term $I I I$, we write

$$
\begin{gathered}
-\int_{\Omega} N\left[M\left[u_{1}\right] \partial_{x} N\left[u_{1}\right]\right] N\left[u_{1}\right]=-\int_{\Omega} M\left[u_{1}\right] \partial_{x} N\left[u_{1}\right] N\left[u_{1}\right] \\
\quad \leq \int_{0}^{L_{1}} \mid M\left[u_{1}\right]\left\|\partial_{x} N\left[u_{1}\right]\right\|_{L_{y}^{2}\left(\left[0, L_{2}\right]\right)}\left\|N\left[u_{1}\right]\right\|_{L_{y}^{2}\left(\left[0, L_{2}\right]\right)} d x \\
\quad \leq\left\|M\left[u_{1}\right]\right\|_{L_{x}^{2}\left(\left[0, L_{1}\right]\right)}\left\|\partial_{x} N\left[u_{1}\right]\right\|_{L^{2}(\Omega)}\left\|N\left[u_{1}\right]\right\|_{L_{y}^{2} L_{x}^{\infty}(\Omega)} .
\end{gathered}
$$

Upon (5.22) we can use Agmon's inequality in the $x$-variable thus the far right side of (5.35) can be estimated by

$$
C\left\|M\left[u_{1}\right]\right\|_{L_{x}^{2}\left(\left[0, L_{1}\right]\right)}\left\|\partial_{x} N\left[u_{1}\right]\right\|_{L^{2}(\Omega)}\left\|N\left[u_{1}\right]\right\|_{L^{2}(\Omega)}^{1 / 2}\left\|\partial_{x} N\left[u_{1}\right]\right\|_{L^{2}(\Omega)}^{1 / 2} .
$$

The above expression equals

$$
\begin{aligned}
& C L_{2}^{-1 / 2}\left\|M\left[u_{1}\right]\right\|_{L^{2}(\Omega)} L_{2}\left\|\partial_{x y} N\left[u_{1}\right]\right\|_{L^{2}(\Omega)} L_{2}\left\|\partial_{y y} N\left[u_{1}\right]\right\|_{L^{2}(\Omega)}^{1 / 2} L_{2}^{1 / 2}\left\|\partial_{x y} N\left[u_{1}\right]\right\|_{L^{2}(\Omega)}^{1 / 2} \\
& \quad \leq C L_{2}^{2}\left\|M\left[u_{1}\right]\right\|_{L^{2}(\Omega)}\left\|\Delta N\left[u_{1}\right]\right\|_{L^{2}(\Omega)}^{2} \\
& \quad \leq C L_{2}^{2}\left(\sqrt{F\left(u_{1}\right)}+\|\Phi\|_{L^{2}(\Omega)}\right)\left\|\Delta N\left[u_{1}\right]\right\|_{L^{2}(\Omega)}^{2} .
\end{aligned}
$$

Regarding the term $I V$, we have

$$
-\int_{\Omega} N\left[N\left[u_{1}\right] \partial_{x} M\left[u_{1}\right]\right] N\left[u_{1}\right]=-\int_{\Omega} N\left[u_{1}\right] \partial_{x} M\left[u_{1}\right] N\left[u_{1}\right]=2 \int_{\Omega} \partial_{x} N\left[u_{1}\right] M\left[u_{1}\right] N\left[u_{1}\right] .
$$

Estimates (5.35)-(5.37) yield

$$
I V \leq C L_{2}^{2}\left(\sqrt{F\left(u_{1}\right)}+\|\Phi\|_{L^{2}(\Omega)}\right)\left\|\Delta N\left[u_{1}\right]\right\|_{L^{2}(\Omega)}^{2} .
$$


We turn to the second term on the right side of (5.31). Due to (5.21), we have

$$
-\int_{\Omega} N\left[u_{2} \partial_{x} u_{2}\right] N\left[u_{1}\right]=-\int_{\Omega} N\left[N\left[u_{2}\right] \partial_{x} N\left[u_{2}\right]\right] N\left[u_{1}\right]=-\int_{\Omega} N\left[u_{2}\right] \partial_{x} N\left[u_{2}\right] N\left[u_{1}\right] .
$$

In order to estimate the right side of (5.40), we note that from $(1.2)_{3}$

$$
\begin{aligned}
& -\int_{\Omega} N\left[u_{2}\right] \partial_{x} N\left[u_{2}\right] N\left[u_{1}\right]=-\int_{\Omega} N\left[u_{2}\right] \partial_{y} N\left[u_{1}\right] N\left[u_{1}\right] \\
& \quad \leq \int_{0}^{L_{1}}\left\|u_{2}\right\|_{L_{y}^{2}\left(\left[0, L_{2}\right]\right)}\left\|\partial_{y} N\left[u_{1}\right]\right\|_{L_{y}^{2}\left(\left[0, L_{2}\right]\right)}\left\|N\left[u_{1}\right]\right\|_{L_{y}^{\infty}\left(\left[0, L_{2}\right]\right)} d x \\
& \quad \leq C \int_{0}^{L_{1}}\left\|u_{2}\right\|_{L_{y}^{2}\left(\left[0, L_{2}\right]\right)}\left\|\partial_{y} N\left[u_{1}\right]\right\|_{L_{y}^{2}\left(\left[0, L_{2}\right]\right)}\left\|N\left[u_{1}\right]\right\|_{L_{y}^{2}\left(\left[0, L_{2}\right]\right)}^{1 / 2}\left\|\partial_{y} N\left[u_{1}\right]\right\|_{L_{y}^{2}\left(\left[0, L_{2}\right]\right)}^{1 / 2} d x \\
& \quad \leq C\left\|u_{2}\right\|_{L^{2}(\Omega)}\left\|\partial_{y} N\left[u_{1}\right]\right\|_{L_{y}^{2} L_{x}^{\infty}(\Omega)}\left\|N\left[u_{1}\right]\right\|_{L^{2}(\Omega)}^{1 / 2}\left\|\partial_{y} N\left[u_{1}\right]\right\|_{L^{2}(\Omega)}^{1 / 2},
\end{aligned}
$$

where we used Agmon's inequality in the $y$-variable and Hölder's inequality. By Agmon's inequality in the $x$-variable, since $u_{1}=N_{x}\left[u_{1}\right]$, we may bound the right side of (5.41) by

$$
C\left\|u_{2}\right\|_{L^{2}(\Omega)}\left\|\partial_{y} N\left[u_{1}\right]\right\|_{L^{2}(\Omega)}^{1 / 2}\left\|\partial_{y x} N\left[u_{1}\right]\right\|_{L^{2}(\Omega)}^{1 / 2}\left\|N\left[u_{1}\right]\right\|_{L^{2}(\Omega)}^{1 / 2}\left\|\partial_{y} N\left[u_{1}\right]\right\|_{L^{2}(\Omega)}^{1 / 2},
$$

Using Lemma 4.3 we estimate the above expression by

$$
\begin{aligned}
& C\left\|u_{2}\right\|_{L^{2}(\Omega)} L_{2}^{1 / 2}\left\|\partial_{y y} N\left[u_{1}\right]\right\|_{L^{2}(\Omega)}^{1 / 2}\left\|\partial_{y x} N\left[u_{1}\right]\right\|_{L^{2}(\Omega)}^{1 / 2} L_{2}\left\|\partial_{y y} N\left[u_{1}\right]\right\|_{L^{2}(\Omega)}^{1 / 2} L_{2}^{1 / 2}\left\|\partial_{y y} N\left[u_{1}\right]\right\|_{L^{2}(\Omega)}^{1 / 2} \\
& \quad \leq C L_{2}^{2}\left\|u_{2}\right\|_{L^{2}(\Omega)}\left\|\Delta N\left[u_{1}\right]\right\|_{L^{2}(\Omega)}^{2} .
\end{aligned}
$$

Estimates (5.31)-(5.43) give

$$
\begin{aligned}
& \frac{1}{2} \frac{d}{d t}\left\|N\left[u_{1}\right]\right\|_{L^{2}(\Omega)}^{2}+\left\|\Delta N\left[u_{1}\right]\right\|_{L^{2}(\Omega)}^{2}-\left\|\nabla N\left[u_{1}\right]\right\|_{L^{2}(\Omega)}^{2} \\
& \quad \leq C L_{2}^{2}\left(\sqrt{F\left(u_{1}\right)}+\|\Phi\|_{L^{2}(\Omega)}+\left\|u_{2}\right\|_{L^{2}(\Omega)}\right)\left\|\Delta N\left[u_{1}\right]\right\|_{L^{2}(\Omega)}^{2} .
\end{aligned}
$$

Lemma 4.3 yields

$$
\left\|\nabla N\left[u_{1}\right]\right\|_{L_{2}(\Omega)}^{2} \leq C L_{2}^{2}\left\|\nabla \partial_{y} N\left[u_{1}\right]\right\|_{L^{2}(\Omega)}^{2} \leq C L_{2}^{2}\left\|\Delta N\left[u_{1}\right]\right\|_{L^{2}(\Omega)}^{2} .
$$

Therefore, estimate (5.44) leads to

$$
\begin{aligned}
& \frac{1}{2} \frac{d}{d t}\left\|N\left[u_{1}\right]\right\|_{L^{2}(\Omega)}^{2}+\frac{1}{2}\left\|\Delta N\left[u_{1}\right]\right\|_{L^{2}(\Omega)}^{2} \\
& \quad \leq-\left(\frac{1}{2}-C L_{2}^{2}-C L_{2}^{2}\left(\sqrt{F\left(u_{1}\right)}+\|\Phi\|_{L^{2}(\Omega)}+\left\|u_{2}\right\|_{L^{2}(\Omega)}\right)\right)\left\|\Delta N\left[u_{1}\right]\right\|_{L^{2}(\Omega)}^{2} .
\end{aligned}
$$

In order to close estimates (5.30) and (5.46), we need to bound $F\left(u_{1}\right)$. We fix $t_{0}>0$ in $\left[0, T_{*}\right)$, the maximal interval of existence of $u$, and we set $v_{1}(t)=M\left[u_{1}\right](t)-\Phi_{b^{*}\left(t_{0}\right)}=M\left[u_{1}\right](t)-\Phi_{b^{*}}$. Applying the operator $M[\cdot]$ to the equation $(1.2)_{1}$, we note that $v_{1}$ satisfies the equation

$$
\partial_{t} v_{1}+\left(\partial_{x x x x}+\partial_{x x}\right) v_{1}+M\left[u_{1} \partial_{x} u_{1}\right]+M\left[u_{2} \partial_{x} u_{2}\right]=-\left(\partial_{x x x x}+\partial_{x x}\right) \Phi_{b^{*}}
$$


Multiplying (5.47) by $v_{1}$ and integrating by parts over $\Omega$, we get at time $t_{0}$

$$
\begin{aligned}
& \left.\frac{1}{2} \frac{d}{d t}\left\|v_{1}\right\|_{L^{2}(\Omega)}^{2}\right|_{t=t_{0}}+\left\|\partial_{x x} v_{1}\right\|_{L^{2}(\Omega)}^{2}-\left\|\partial_{x} v_{1}\right\|_{L^{2}(\Omega)}^{2}+\int_{\Omega} M\left[u_{1} \partial_{x} u_{1}\right] v_{1}+\int_{\Omega} M\left[u_{2} \partial_{x} u_{2}\right] v_{1} \\
& =-\int_{\Omega}\left(\partial_{x x x x}+\partial_{x x}\right) \Phi_{b^{*}} v_{1} .
\end{aligned}
$$

Regarding the fourth term on the left side of $(5.48)$, we note that since $v_{1}=M\left[v_{1}\right]$, we obtain

$$
\begin{aligned}
\int_{\Omega} M\left[u_{1} \partial_{x} u_{1}\right] v_{1}= & \int_{\Omega} M\left[N\left[u_{1}\right] \partial_{x} N\left[u_{1}\right]\right] M\left[v_{1}\right]+\int_{\Omega} M\left[M\left[u_{1}\right] \partial_{x} M\left[u_{1}\right]\right] M\left[v_{1}\right] \\
& +\int_{\Omega} M\left[N\left[u_{1}\right] \partial_{x} M\left[u_{1}\right]\right] M\left[v_{1}\right]+\int_{\Omega} M\left[M\left[u_{1}\right] \partial_{x} N\left[u_{1}\right]\right] M\left[v_{1}\right] \\
= & \int_{\Omega} N\left[u_{1}\right] \partial_{x} N\left[u_{1}\right] M\left[v_{1}\right]+\int_{\Omega} M\left[u_{1}\right] \partial_{x} M\left[u_{1}\right] M\left[v_{1}\right] \\
& +\int_{\Omega} N\left[u_{1}\right] \partial_{x} M\left[u_{1}\right] M\left[v_{1}\right]+\int_{\Omega} M\left[u_{1}\right] \partial_{x} N\left[u_{1}\right] M\left[v_{1}\right] \\
= & \int_{\Omega} N\left[u_{1}\right] \partial_{x} N\left[u_{1}\right] v_{1}+\int_{\Omega} M\left[u_{1}\right] \partial_{x} M\left[u_{1}\right] v_{1} .
\end{aligned}
$$

Furthermore, integrating by parts we have

$$
\int_{\Omega} M\left[u_{1}\right] \partial_{x} M\left[u_{1}\right] v_{1}=-\frac{1}{2} \int_{\Omega}\left(M\left[u_{1}\right]\right)^{2} \partial_{x} v_{1}=\frac{1}{2} \int_{\Omega}\left(M\left[u_{1}\right]\right)^{2} \partial_{x} \Phi_{b^{*}}=\frac{1}{2} \int_{\Omega} v_{1}^{2} \partial_{x} \Phi_{b^{*}},
$$

where we used $\int_{\Omega} M\left[u_{1}\right] 2 \partial_{x} u_{1}=\int_{\Omega} M\left[u_{1}\right]^{2} \partial_{x} M\left[u_{1}\right]=0$ in the second equality. Therefore, (5.48) can be rewritten as

$$
\begin{array}{r}
\left.\frac{1}{2} \frac{d}{d t}\left\|v_{1}\right\|_{L^{2}(\Omega)}^{2}\right|_{t=t_{0}}+\left\|\partial_{x x} v_{1}\right\|_{L^{2}(\Omega)}^{2}-\left\|\partial_{x} v_{1}\right\|_{L^{2}(\Omega)}^{2}+\frac{1}{2} \int_{\Omega} v_{1}^{2} \partial_{x} \Phi_{b^{*}} \\
=-\int_{\Omega}\left(\partial_{x}^{4}+\partial_{x x}\right) \Phi_{b^{*}} v_{1}-\int_{\Omega} N\left[u_{1}\right] \partial_{x} N\left[u_{1}\right] v_{1}-\int_{\Omega} N\left[u_{2}\right] \partial_{x} N\left[u_{2}\right] v_{1} .
\end{array}
$$

Integrating by parts in the first term on the right side of (5.51) yields

$$
-\int_{\Omega}\left(\partial_{x}^{4}+\partial_{x x}\right) \Phi_{b^{*}} v_{1}=-\int_{\Omega} \partial_{x x} \Phi_{b^{*}} \partial_{x x} v_{1}+\int_{\Omega} \partial_{x} \Phi_{b^{*}} \partial_{x} v_{1}=-\left(v_{1}, \Phi_{b^{*}}\right)_{\Phi_{b^{*}}}+\int_{\Omega} v_{1} \Phi_{b^{*}} \partial_{x} \Phi_{b^{*}}
$$

Hence, we obtain

$$
\begin{gathered}
\left.\frac{1}{2} \frac{d}{d t}\left\|v_{1}\right\|_{L^{2}(\Omega)}^{2}\right|_{t=t_{0}}+\left\|\partial_{x x} v_{1}\right\|_{L^{2}(\Omega)}^{2}-\left\|\partial_{x} v_{1}\right\|_{L^{2}(\Omega)}^{2}+\frac{1}{2} \int_{\Omega} v_{1}^{2} \partial_{x} \Phi_{b^{*}} \\
=-\left(v_{1}, \Phi_{b^{*}}\right)_{\Phi_{b^{*}}}+\int_{\Omega} v_{1} \Phi_{b^{*}} \partial_{x} \Phi_{b^{*}}-\int_{\Omega} N\left[u_{1}\right] \partial_{x} N\left[u_{1}\right] v_{1}-\int_{\Omega} N\left[u_{2}\right] \partial_{x} N\left[u_{2}\right] v_{1} \\
\leq \frac{1}{4}\left(v_{1}, v_{1}\right)_{\Phi_{b^{*}}}+\left(\Phi_{b^{*}}, \Phi_{b^{*}}\right)_{\Phi_{b^{*}}}+\int_{\Omega} v_{1} \Phi_{b^{*}} \partial_{x} \Phi_{b^{*}} \\
-\int_{\Omega} N\left[u_{1}\right] \partial_{x} N\left[u_{1}\right] v_{1}-\int_{\Omega} N\left[u_{2}\right] \partial_{x} N\left[u_{2}\right] v_{1},
\end{gathered}
$$


where we used the Cauchy-Schwarz inequality since $\mathcal{B}_{\Phi_{b^{*}}}(\cdot)$ is a positive quadratic form. Now we use (4.15) with $f=g=v_{1}$ for the first term on the far right side. We thus get

$$
\begin{gathered}
\left.\frac{1}{2} \frac{d}{d t}\left\|v_{1}\right\|_{L^{2}(\Omega)}^{2}\right|_{t=t_{0}}+\frac{3}{4}\left(\left\|\partial_{x x} v_{1}\right\|_{L^{2}(\Omega)}^{2}-\left\|\partial_{x} v_{1}\right\|_{L^{2}(\Omega)}^{2}\right)+\frac{1}{4} \int_{\Omega} v_{1}^{2} \partial_{x} \Phi_{b^{*}} \\
\leq \frac{1}{L_{1}} \int_{0}^{L_{2}}\left(\int_{0}^{L_{1}} v_{1} \partial_{x} \Phi_{b^{*}} d x\right)^{2} d y+\int_{\Omega} v_{1} \Phi_{b^{*}} \partial_{x} \Phi_{b^{*}}-\int_{\Omega} N\left[u_{1}\right] \partial_{x} N\left[u_{1}\right] v_{1} \\
\quad-\int_{\Omega} N\left[u_{2}\right] \partial_{x} N\left[u_{2}\right] v_{1}+\left\|\partial_{x x} \Phi_{b^{*}}\right\|_{L^{2}(\Omega)}^{2},
\end{gathered}
$$

where we used $\left(\Phi_{b^{*}}, \Phi_{b^{*}}\right)_{\Phi_{b^{*}}} \leq\left\|\partial_{x x} \Phi_{b^{*}}\right\|_{L^{2}(\Omega)}^{2}$, which follows from (4.15). Therefore, we obtain

$$
\begin{gathered}
\left.\frac{1}{2} \frac{d}{d t}\left\|v_{1}\right\|_{L^{2}(\Omega)}^{2}\right|_{t=t_{0}}+\frac{3}{4} \mathcal{B}_{\frac{1}{3} \Phi_{b^{*}}}\left(v_{1}\right) \leq \frac{4}{3 L_{1}} \int_{0}^{L_{2}}\left(\int_{0}^{L_{1}} v_{1} \partial_{x} \Phi_{b^{*}} d x\right)^{2} d y+\int_{\Omega} v_{1} \Phi_{b^{*}} \partial_{x} \Phi_{b^{*}} \\
-\int_{\Omega} N\left[u_{1}\right] \partial_{x} N\left[u_{1}\right] v_{1}-\int_{\Omega} N\left[u_{2}\right] \partial_{x} N\left[u_{2}\right] v_{1}+\left\|\partial_{x x} \Phi_{b^{*}}\right\|_{L^{2}(\Omega)}^{2}
\end{gathered}
$$

Regarding the first term on the right side of (5.55) we note that by (4.13) we have

$$
\int_{\Omega} v_{1}\left(t_{0}\right) \partial_{x} \Phi_{b^{*}\left(t_{0}\right)}=0
$$

Since the function $v_{1}\left(t_{0}\right) \partial_{x} \Phi_{b^{*}\left(t_{0}\right)}$ depends only on the $x$-variable, we obtain

$$
\int_{0}^{L_{1}} v_{1}(x) \partial_{x} \Phi_{b^{*}} d x=0
$$

In order to estimate the second term on the right side of (5.55), we note that

$$
\begin{aligned}
\int_{\Omega} v_{1} & \Phi_{b^{*}} \partial_{x} \Phi_{b^{*}}=L_{2} \int_{0}^{L_{1}} v_{1} \Phi_{b^{*}} \partial_{x} \Phi_{b^{*}} \leq L_{2}\left\|v_{1}\right\|_{L^{2}\left(\left[0, L_{1}\right]\right)}\left\|\Phi_{b^{*}}\right\|_{L^{2}\left(\left[0, L_{1}\right]\right)}\left\|\partial_{x} \Phi_{b^{*}}\right\|_{L^{\infty}\left(\left[0, L_{1}\right]\right)} \\
& \leq L_{2}\left\|v_{1}\right\|_{L^{2}\left(\left[0, L_{1}\right]\right)}\left\|\Phi_{b^{*}}\right\|_{L^{2}\left(\left[0, L_{1}\right]\right)}\left\|\partial_{x} \Phi_{b^{*}}\right\|_{L^{2}\left(\left[0, L_{1}\right]\right)}^{1 / 2}\left\|\partial_{x} x \Phi_{b^{*}}\right\|_{L^{2}\left(\left[0, L_{1}\right]\right)}^{1 / 2} \\
& =C L_{2}^{-1 / 2}\left\|v_{1}\right\|_{L^{2}(\Omega)}\left\|\Phi_{b^{*}}\right\|_{L^{2}(\Omega)}\left\|\partial_{x} \Phi_{b^{*}}\right\|_{L^{2}(\Omega)}^{1 / 2}\left\|\partial_{x x} \Phi_{b^{*}}\right\|_{L^{2}(\Omega)}^{1 / 2} \\
& \leq C L_{2}^{-1 / 2}\left\|v_{1}\right\|_{L^{2}(\Omega)}\left\|\Phi_{b^{*}}\right\|_{L^{2}(\Omega)}^{5 / 4}\left\|\partial_{x x} \Phi_{b^{*}}\right\|_{L^{2}(\Omega)}^{3 / 4}
\end{aligned}
$$

where we used Agmon's inequality and the interpolation inequality. Regarding the third term on the right side of (5.55) we have

$$
\begin{aligned}
& -\int_{\Omega} N\left[u_{1}\right] \partial_{x} N\left[u_{1}\right] v_{1} \leq \int_{0}^{L_{1}}\left\|N\left[u_{1}\right]\right\|_{L_{y}^{2}\left(\left[0, L_{2}\right]\right)}\left\|\partial_{x} N\left[u_{1}\right]\right\|_{L_{y}^{2}\left(\left[0, L_{2}\right]\right)}\left|v_{1}\right| d x \\
& \quad \leq\left\|N\left[u_{1}\right]\right\|_{L^{2}(\Omega)}\left\|\partial_{x} N\left[u_{1}\right]\right\|_{L_{y}^{2} L_{x}^{\infty}(\Omega)}\left\|v_{1}\right\|_{L^{2}\left(\left[0, L_{1}\right]\right)} \\
& \quad \leq C\left\|N\left[u_{1}\right]\right\|_{L^{2}(\Omega)}\left\|\partial_{x} N\left[u_{1}\right]\right\|_{L^{2}(\Omega)}^{1 / 2}\left\|\partial_{x x} N\left[u_{1}\right]\right\|_{L^{2}(\Omega)}^{1 / 2} L_{2}^{-1 / 2}\left\|v_{1}\right\|_{L^{2}(\Omega)},
\end{aligned}
$$

where we used Agmon's inequality. Applying Lemma 4.3, we may bound the above expression by

$$
\begin{gathered}
C L_{2}^{2}\left\|\partial_{y y} N\left[u_{1}\right]\right\|_{L^{2}(\Omega)} L_{2}^{1 / 2}\left\|\partial_{y x} N\left[u_{1}\right]\right\|^{1 / 2}\left\|\partial_{x x} N\left[u_{1}\right]\right\|^{1 / 2} L_{2}^{-1 / 2}\left\|v_{1}\right\|_{L^{2}(\Omega)} \\
\leq C L_{2}^{2}\left\|v_{1}\right\|_{L^{2}(\Omega)}\left\|\Delta N\left[u_{1}\right]\right\|_{L^{2}(\Omega)}^{2} .
\end{gathered}
$$


In order to estimate the fourth term on the right side of (5.55) we proceed in a similar way and obtain

$$
-\int_{\Omega} N\left[u_{2}\right] \partial_{x} N\left[u_{2}\right] v_{1} \leq C L_{2}^{2}\left\|v_{1}\right\|_{L^{2}(\Omega)}\left\|\Delta u_{2}\right\|_{L^{2}(\Omega)}^{2}
$$

Estimates (5.55)-(5.61) combined with (4.17) lead to

$$
\begin{aligned}
& \left.\frac{1}{2} \frac{d}{d t}\left\|v_{1}\right\|_{L^{2}(\Omega)}^{2}\right|_{t=t_{0}}+\left\|v_{1}\right\|_{L^{2}(\Omega)}^{2} \\
& \leq C L_{2}^{-1 / 2}\left\|v_{1}\right\|_{L^{2}(\Omega)}\left\|\Phi_{b^{*}}\right\|_{L^{2}(\Omega)}^{5 / 4}\left\|\partial_{x x} \Phi_{b^{*}}\right\|_{L^{2}(\Omega)}^{3 / 4}+C L_{2}^{2}\left\|v_{1}\right\|_{L^{2}(\Omega)}\left\|\Delta N\left[u_{1}\right]\right\|_{L^{2}(\Omega)}^{2} \\
& \quad+C L_{2}^{2}\left\|v_{1}\right\|_{L^{2}(\Omega)}\left\|\Delta u_{2}\right\|_{L^{2}(\Omega)}^{2}+\left\|\partial_{x x} \Phi_{b^{*}}\right\|_{L^{2}(\Omega)}^{2}
\end{aligned}
$$

from which we obtain

$$
\begin{aligned}
\left.\frac{1}{2} \frac{d}{d t}\left\|v_{1}\right\|_{L^{2}(\Omega)}^{2}\right|_{t=t_{0}} \leq- & \frac{1}{2}\left\|v_{1}\right\|_{L^{2}(\Omega)}^{2}+C L_{2}^{-1}\left\|\Phi_{b^{*}}\right\|_{L^{2}(\Omega)}^{5 / 2}\left\|\partial_{x x} \Phi_{b^{*}}\right\|_{L^{2}(\Omega)}^{3 / 2}+\left\|\partial_{x x} \Phi_{b^{*}}\right\|_{L^{2}(\Omega)}^{2} \\
& +C L_{2}^{2}\left\|v_{1}\right\|_{L^{2}(\Omega)}\left\|\Delta N\left[u_{1}\right]\right\|_{L^{2}(\Omega)}^{2}+C L_{2}^{2}\left\|v_{1}\right\|_{L^{2}(\Omega)}\left\|\Delta u_{2}\right\|_{L^{2}(\Omega)}^{2}
\end{aligned}
$$

Therefore, by (4.14), we have

$$
\begin{aligned}
\frac{1}{2} \frac{d}{d t} F\left(u_{1}\right) \leq- & \frac{1}{2} F\left(u_{1}\right)+C L_{2}^{-1}\left\|\Phi_{b^{*}}\right\|_{L^{2}(\Omega)}^{5 / 2}\left\|\partial_{x x} \Phi_{b^{*}}\right\|_{L^{2}(\Omega)}^{3 / 2}+\left\|\partial_{x x} \Phi_{b^{*}}\right\|_{L^{2}(\Omega)}^{2} \\
& +C L_{2}^{2} \sqrt{F\left(u_{1}\right)}\left\|\Delta N\left[u_{1}\right]\right\|_{L^{2}(\Omega)}^{2}+C L_{2}^{2} \sqrt{F\left(u_{1}\right)}\left\|\Delta u_{2}\right\|_{L^{2}(\Omega)}^{2}
\end{aligned}
$$

In order to conclude the proof of the theorem, we gather inequalities (5.30), (5.46), and (5.64). The quantities $\left\|u_{2}\right\|_{L^{2}(\Omega)},\left\|N\left[u_{1}\right]\right\|_{L^{2}(\Omega)}$ and $F\left(u_{1}\right)$ satisfy the estimates

$$
\begin{gathered}
\frac{1}{2} \frac{d}{d t}\left\|u_{2}\right\|_{L^{2}(\Omega)}^{2}+\frac{1}{2}\left\|\Delta u_{2}\right\|_{L^{2}(\Omega)}^{2} \\
\leq-\left(\frac{1}{2}-\left(\frac{L_{2}}{2 \pi}\right)^{2}-C L_{2}^{2}\left(\|\Phi\|_{L^{2}(\Omega)}+\left\|N\left[u_{1}\right]\right\|_{L^{2}(\Omega)}+\sqrt{F\left(u_{1}\right)}\right)\right)\left\|\Delta u_{2}\right\|_{L^{2}(\Omega)}^{2} \\
\frac{1}{2} \frac{d}{d t}\left\|N\left[u_{1}\right]\right\|_{L^{2}(\Omega)}^{2}+\frac{1}{2}\left\|\Delta N\left[u_{1}\right]\right\|_{L^{2}(\Omega)}^{2} \\
\leq-\left(\frac{1}{2}-C L_{2}^{2}-C L_{2}^{2}\left(\sqrt{F\left(u_{1}\right)}+\|\Phi\|_{L^{2}(\Omega)}+\left\|u_{2}\right\|_{L^{2}(\Omega)}\right)\right)\left\|\Delta N\left[u_{1}\right]\right\|_{L^{2}(\Omega)}^{2} \\
\frac{1}{2} \frac{d}{d t} F\left(u_{1}\right) \leq-\frac{1}{2} F\left(u_{1}\right)+C L_{2}^{-1}\left\|\Phi_{b^{*}}\right\|_{L^{2}(\Omega)}^{5 / 2}\left\|\partial_{x x} \Phi_{b^{*}}\right\|_{L^{2}(\Omega)}^{3 / 2}+\left\|\partial_{x x} \Phi_{b^{*}}\right\|_{L^{2}(\Omega)}^{2} \\
\quad+C L_{2}^{2} \sqrt{F\left(u_{1}\right)}\left\|\Delta N\left[u_{1}\right]\right\|_{L^{2}(\Omega)}^{2}+C L_{2}^{2} \sqrt{F\left(u_{1}\right)}\left\|\Delta u_{2}\right\|_{L^{2}(\Omega)}^{2} .
\end{gathered}
$$


Using (4.18) and (4.19) we may restate the above inequalities as

$$
\begin{aligned}
& \frac{1}{2} \frac{d}{d t}\left\|u_{2}\right\|_{L^{2}(\Omega)}^{2}+\frac{1}{2}\left\|\Delta u_{2}\right\|_{L^{2}(\Omega)}^{2} \\
& \leq-\left(\frac{1}{2}-\left(\frac{L_{2}}{2 \pi}\right)^{2}-C_{1} L_{2}^{2}\left(L_{1}^{4 / 5} L_{2}^{1 / 2}+\left\|N\left[u_{1}\right]\right\|_{L^{2}(\Omega)}+\sqrt{F\left(u_{1}\right)}\right)\right)\left\|\Delta u_{2}\right\|_{L^{2}(\Omega)}^{2} \\
& \frac{1}{2} \frac{d}{d t}\left\|N\left[u_{1}\right]\right\|_{L^{2}(\Omega)}^{2}+\frac{1}{2}\left\|\Delta N\left[u_{1}\right]\right\|_{L^{2}(\Omega)}^{2} \\
& \leq-\left(\frac{1}{2}-C_{1} L_{2}^{2}-C_{1} L_{2}^{2}\left(L_{1}^{4 / 5} L_{2}^{1 / 2}+\sqrt{F\left(u_{1}\right)}+\left\|u_{2}\right\|_{L^{2}(\Omega)}\right)\right)\left\|\Delta N\left[u_{1}\right]\right\|_{L^{2}(\Omega)}^{2} \\
& \frac{1}{2} \frac{d}{d t} F\left(u_{1}\right) \leq-\frac{1}{2} F\left(u_{1}\right)+C_{1} L_{2}^{2} \sqrt{F\left(u_{1}\right)}\left\|\Delta N\left[u_{1}\right]\right\|_{L^{2}(\Omega)}^{2}+C_{1} L_{2}^{2} \sqrt{F\left(u_{1}\right)}\left\|\Delta u_{2}\right\|_{L^{2}(\Omega)}^{2} \\
& +C_{1} L_{1}^{16 / 5} L_{2}+C_{1} L_{1}^{22 / 5} L_{2} \text {. }
\end{aligned}
$$

We claim that if $u(t)$ satisfies

$$
F\left(u_{1}\right)(0)+\left\|N\left[u_{1}\right](0)\right\|_{L^{2}(\Omega)}^{2}+\left\|u_{2}(0)\right\|_{L^{2}(\Omega)}^{2}<C_{3}^{-2} L_{2}^{-4},
$$

then

$$
F\left(u_{1}\right)(t)+\left\|N\left[u_{1}\right](t)\right\|_{L^{2}(\Omega)}^{2}+\left\|u_{2}(t)\right\|_{L^{2}(\Omega)}^{2}<C_{3}^{-2} L_{2}^{-4},
$$

for a certain constant $C_{3}$ which is to be determined and for all $t \in\left[0, T_{*}\right)$, where $T_{*}$ is the maximal time of existence in Proposition 4.1. For the sake of obtaining a contradiction, suppose that there exists $t_{1} \in\left(0, T_{*}\right)$ such that $(5.68)$ holds for any $t \in\left[0, t_{1}\right)$ and

$$
F\left(u_{1}\right)\left(t_{1}\right)+\left\|N\left[u_{1}\right]\left(t_{1}\right)\right\|_{L^{2}(\Omega)}^{2}+\left\|u_{2}\left(t_{1}\right)\right\|_{L^{2}(\Omega)}^{2}=C_{3}^{-2} L_{2}^{-4},
$$

Note, that (5.67) implies that each of the quantities $\sqrt{F\left(u_{1}\right)(0)},\left\|N\left[u_{1}\right](0)\right\|_{L^{2}(\Omega)}$, and $\left\|u_{2}(0)\right\|_{L^{2}(\Omega)}$ is bounded by $C_{3}^{-1} L_{2}^{-2}$. Assuming that

$$
L_{2} \leq C_{3}^{-1} L_{1}^{-22 / 25}
$$

we obtain for $t \in\left[0, t_{1}\right)$

$$
\begin{aligned}
-\frac{1}{4} & +C_{1} L_{2}^{2}\left(C_{2} L_{1}^{4 / 5} L_{2}^{1 / 2}+\left\|N\left[u_{1}\right]\right\|_{L^{2}(\Omega)}+\sqrt{F\left(u_{1}\right)}+\left\|u_{2}\right\|_{L^{2}(\Omega)}\right) \\
& \leq-\frac{1}{4}+C_{1} C_{2} C_{3}^{-5 / 2} L_{1}^{-7 / 5}+3 C_{1} C_{3}^{-1} \leq 0
\end{aligned}
$$

where we used (5.68) and (5.70) in the first inequality and assumed that $C_{3}$ is a large enough constant in the second. Using $(5.71)$ in $(5.66)_{1}$ and $(5.66)_{2}$, we have on $\left(0, t_{1}\right)$

$$
\begin{aligned}
& \frac{1}{2} \frac{d}{d t}\left\|u_{2}\right\|_{L^{2}(\Omega)}^{2}+\frac{1}{2}\left\|\Delta u_{2}\right\|_{L^{2}(\Omega)}^{2} \leq-\left(\frac{1}{4}-\left(\frac{L_{2}}{2 \pi}\right)^{2}\right)\left\|\Delta u_{2}\right\|_{L^{2}(\Omega)}^{2}, \\
& \frac{1}{2} \frac{d}{d t}\left\|N\left[u_{1}\right]\right\|_{L^{2}(\Omega)}^{2}+\frac{1}{2}\left\|\Delta N\left[u_{1}\right]\right\|_{L^{2}(\Omega)}^{2} \leq-\left(\frac{1}{4}-C_{1} L_{2}^{2}\right)\left\|\Delta N\left[u_{1}\right]\right\|_{L^{2}(\Omega)}^{2} .
\end{aligned}
$$


Therefore the quantities $\left\|N\left[u_{1}\right]\right\|_{L^{2}(\Omega)}$ and $\left\|u_{2}\right\|_{L^{2}(\Omega)}$ are non-increasing on $\left[0, t_{1}\right]$. Furthermore, adding $(5.66)_{3},(5.72)_{1}$, and $(5.72)_{2}$, and using (5.72) we obtain

$$
\begin{aligned}
\frac{1}{2} \frac{d}{d t}( & \left.F\left(u_{1}\right)+\left\|N\left[u_{1}\right]\right\|_{L^{2}(\Omega)}^{2}+\left\|u_{2}\right\|_{L^{2}(\Omega)}^{2}\right)+\frac{1}{2}\left\|\Delta N\left[u_{1}\right]\right\|_{L^{2}(\Omega)}^{2}+\frac{1}{2}\left\|\Delta u_{2}\right\|_{L^{2}(\Omega)}^{2} \\
\leq & -\left(\frac{1}{4}-\left(\frac{L_{2}}{2 \pi}\right)^{2}\right)\left\|\Delta u_{2}\right\|_{L^{2}(\Omega)}^{2}-\left(\frac{1}{4}-C_{1} L_{2}^{2}\right)\left\|\Delta N\left[u_{1}\right]\right\|_{L^{2}(\Omega)}^{2} \\
& -\frac{1}{2} F\left(u_{1}\right)+C_{1} L_{2}^{2} \sqrt{F\left(u_{1}\right)}\left\|\Delta N\left[u_{1}\right]\right\|_{L^{2}(\Omega)}^{2}+C_{1} L_{2}^{2} \sqrt{F\left(u_{1}\right)}\left\|\Delta u_{2}\right\|_{L^{2}(\Omega)}^{2} \\
& +C_{1} L_{1}^{16 / 5} L_{2}+C_{1} L_{1}^{22 / 5} L_{2} \\
\leq & -\left(\frac{1}{4}-\left(\frac{L_{2}}{2 \pi}\right)^{2}\right)\left\|\Delta u_{2}\right\|_{L^{2}(\Omega)}^{2}-\left(\frac{1}{4}-C_{1} L_{2}^{2}\right)\left\|\Delta N\left[u_{1}\right]\right\|_{L^{2}(\Omega)}^{2} \\
& \quad-\frac{1}{2} F\left(u_{1}\right)+C_{1} L_{2}^{2}\left(C_{3}^{-1} L_{2}^{-2}\right)\left\|\Delta N\left[u_{1}\right]\right\|_{L^{2}(\Omega)}^{2}+C_{1} L_{2}^{2}\left(C_{3}^{-1} L_{2}^{-2}\right)\left\|\Delta u_{2}\right\|_{L^{2}(\Omega)}^{2} \\
& +C_{1} L_{1}^{16 / 5} L_{2}+C_{1} L_{1}^{22 / 5} L_{2}
\end{aligned}
$$

Possibly making $C_{3}$ larger and $L_{2}$ small, we may assume that

$$
\frac{1}{4}-\max \left\{1, C_{1}\right\} L_{2}^{2}-\frac{C_{1}}{C_{3}} \geq 0,
$$

(note that $L_{2} \leq 1 / 2 \pi$ by (5.70)). Estimate (5.73) leads to

$$
\begin{aligned}
\frac{1}{2} \frac{d}{d t} & \left(F\left(u_{1}\right)+\left\|N\left[u_{1}\right]\right\|_{L^{2}(\Omega)}^{2}+\left\|u_{2}\right\|_{L^{2}(\Omega)}^{2}\right)+\frac{1}{2}\left\|\Delta N\left[u_{1}\right]\right\|_{L^{2}(\Omega)}^{2}+\frac{1}{2}\left\|\Delta u_{2}\right\|_{L^{2}(\Omega)}^{2} \\
& \leq-\frac{1}{2} F\left(u_{1}\right)+C_{1} L_{1}^{16 / 5} L_{2}+C_{1} L_{1}^{22 / 5} L_{2} .
\end{aligned}
$$

The Poincaré inequalities

$$
\left\|N\left[u_{1}\right]\right\|_{L^{2}(\Omega)}^{2} \leq\left(\frac{L_{2}}{2 \pi}\right)^{2}\left\|\nabla N\left[u_{1}\right]\right\|_{L^{2}(\Omega)}^{2} \leq\left(\frac{L_{2}}{2 \pi}\right)^{4}\left\|\Delta N\left[u_{1}\right]\right\|_{L^{2}(\Omega)}^{2}
$$

and

$$
\left\|u_{2}\right\|_{L^{2}(\Omega)}^{2} \leq\left(\frac{L_{2}}{2 \pi}\right)^{2}\left\|\nabla u_{2}\right\|_{L^{2}(\Omega)}^{2} \leq\left(\frac{L_{2}}{2 \pi}\right)^{4}\left\|\Delta u_{2}\right\|_{L^{2}(\Omega)}^{2}
$$

applied to the second and third term on the left side of (5.75), combined with $L_{2} \leq 2 \pi$, yield

$$
\begin{aligned}
& \frac{1}{2} \frac{d}{d t}\left(F\left(u_{1}\right)+\left\|N\left[u_{1}\right]\right\|_{L^{2}(\Omega)}^{2}+\left\|u_{2}\right\|_{L^{2}(\Omega)}^{2}\right) \\
& \quad \leq-\frac{1}{2}\left(F\left(u_{1}\right)+\left\|N\left[u_{1}\right]\right\|_{L^{2}(\Omega)}^{2}+\left\|u_{2}\right\|_{L^{2}(\Omega)}^{2}\right)+C_{1} L_{1}^{16 / 5} L_{2}+C_{1} L_{1}^{22 / 5} L_{2} .
\end{aligned}
$$

At time $t=t_{1}$, due to assumption (5.69) we obtain

$$
\left.\frac{d}{d t}\left(F\left(u_{1}\right)+\left\|N\left[u_{1}\right]\right\|_{L^{2}(\Omega)}^{2}+\left\|u_{2}\right\|_{L^{2}(\Omega)}^{2}\right)\right|_{t=t_{1}} \leq-C_{3}^{-2} L_{2}^{-4}+C_{1} L_{1}^{16 / 5} L_{2}+C_{1} L_{1}^{22 / 5} L_{2} .
$$

But by (5.70) we have

$$
2 C_{1} L_{1}^{16 / 5} L_{2}+2 C_{1} L_{1}^{22 / 5} L_{2}<C_{3}^{-2} L_{2}^{-4}
$$


if $C_{3}$ is large enough, which implies

$$
\left.\frac{d}{d t}\left(F\left(u_{1}\right)+\left\|N\left[u_{1}\right]\right\|_{L^{2}(\Omega)}^{2}+\left\|u_{2}\right\|_{L^{2}(\Omega)}^{2}\right)\right|_{t=t_{1}}<0 .
$$

This however is impossible since the definition of $t_{1}$ implies that this derivative is nonnegative.

We note that due to $(5.70)$

$$
\|\Phi\|_{L^{2}(\Omega)} \leq C L_{1}^{4 / 5} L_{2}^{1 / 2}<C C_{3}^{-5 / 2} L_{1}^{-7 / 5} L_{2}^{-2} .
$$

Hence (5.67) is satisfied provided

$$
\begin{gathered}
\left\|M\left[u_{1}\right](0)\right\|_{L^{2}(\Omega)} \leq C_{0}^{-1} L_{2}^{-2}, \\
\left\|N\left[u_{1}\right](0)\right\|_{L^{2}(\Omega)} \leq C_{0}^{-1} L_{2}^{-2},
\end{gathered}
$$

and

$$
\left\|u_{2}(0)\right\|_{L^{2}(\Omega)} \leq C_{0}^{-1} L_{2}^{-2},
$$

for sufficiently large $C_{0}>1$. Therefore Theorem 3.1 is proven.

Remark 5.1. We note that the previous estimates imply the bounds

$$
\begin{aligned}
& \left\|u_{1}(t)\right\|_{L^{2}(\Omega)}^{2} \leq C_{0}^{-2} L_{2}^{-4}\left(e^{-\beta t}+e^{-t}\right)+2\left(C_{1} L_{1}^{16 / 5} L_{2}+C_{1} L_{1}^{22 / 5} L_{2}\right)+C L_{1}^{8 / 5} L_{2} \\
& \left\|u_{2}(t)\right\|_{L^{2}(\Omega)}^{2} \leq\left\|u_{2}(0)\right\|_{L^{2}(\Omega)}^{2} e^{-\left(3 / 2-L_{2}^{2} / 2 \pi^{2}\right) L_{2}^{4} / 16 \pi^{4}},
\end{aligned}
$$

which hold under the assumptions of Theorem 3.1.

Proof. We note that $(5.72)_{1}$ implies

$$
\frac{1}{2} \frac{d}{d t}\left\|u_{2}\right\|_{L^{2}(\Omega)}^{2}+\left(\frac{3}{4}-\left(\frac{L_{2}}{2 \pi}\right)^{2}\right)\left\|\Delta u_{2}\right\|_{L^{2}(\Omega)}^{2} \leq 0,
$$

which, combined with the Poincaré inequality (5.76), yields

$$
\frac{1}{2} \frac{d}{d t}\left\|u_{2}\right\|_{L^{2}(\Omega)}^{2}+\left(\frac{3}{4}-\left(\frac{L_{2}}{2 \pi}\right)^{2}\right)\left(\frac{L_{2}}{2 \pi}\right)^{4}\left\|u_{2}\right\|_{L^{2}(\Omega)}^{2} \leq 0 .
$$

Letting

$$
\alpha=2\left(\frac{3}{4}-\left(\frac{L_{2}}{2 \pi}\right)^{2}\right)\left(\frac{L_{2}}{2 \pi}\right)^{4}
$$

we get from (5.87)

$$
\left\|u_{2}(t)\right\|_{L^{2}(\Omega)}^{2} \leq\left\|u_{2}(0)\right\|_{L^{2}(\Omega)}^{2} e^{-\alpha t} .
$$

Similarly, from $(5.72)_{2}$, using the Poincaré inequality (5.77), we obtain

$$
\left\|N\left[u_{1}\right](t)\right\|_{L^{2}(\Omega)}^{2} \leq\left\|N\left[u_{1}\right](0)\right\|_{L^{2}(\Omega)}^{2} e^{-\beta t},
$$


where

$$
\beta=2\left(\frac{3}{4}-C_{1} L_{2}^{2}\right)\left(\frac{L_{2}}{2 \pi}\right)^{4}
$$

Let

$$
S(t)=F\left(u_{1}\right)(t)+\left\|N\left[u_{1}\right](t)\right\|_{L^{2}(\Omega)}^{2}+\left\|u_{2}(t)\right\|_{L^{2}(\Omega)}^{2} .
$$

From (5.79), it follows

$$
S(t) \leq S(0) e^{-t}+2\left(C_{1} L_{1}^{16 / 5} L_{2}+C_{1} L_{1}^{22 / 5} L_{2}\right) .
$$

In particular, using (5.67), we have

$$
F\left(u_{1}\right)(t) \leq\left(C_{3}^{-2} L_{2}^{-4}\right) e^{-t}+2\left(C_{1} L_{1}^{16 / 5} L_{2}+C_{1} L_{1}^{22 / 5} L_{2}\right) .
$$

Since

$$
\begin{gathered}
\left\|u_{1}\right\|_{L^{2}(\Omega)}^{2} \leq\left\|N\left[u_{1}\right]\right\|_{L^{2}(\Omega)}^{2}+\left\|M\left[u_{1}\right]-\Phi\right\|_{L^{2}(\Omega)}^{2}+\|\Phi\|_{L^{2}(\Omega)}^{2} \\
\leq\left\|N\left[u_{1}\right]\right\|_{L^{2}(\Omega)}^{2}+F\left(u_{1}\right)+\|\Phi\|_{L^{2}(\Omega)}^{2},
\end{gathered}
$$

we obtain

$$
\left\|u_{1}(t)\right\|_{L^{2}(\Omega)}^{2} \leq C_{3}^{-2} L_{2}^{-4}\left(e^{-\beta t}+e^{-t}\right)+2\left(C_{1} L_{1}^{16 / 5} L_{2}+C_{1} L_{1}^{22 / 5} L_{2}\right)+C L_{1}^{8 / 5} L_{2} .
$$

Therefore the inequalities $(5.85)_{1}$ and $(5.85)_{2}$ are proven.

Acknowledgements. I.K. was partially supported by the NSF grant DMS-1009769, while M.Z. was partially supported by the NSF grant DMS-1109562. M.Z. is grateful to the Institut Élie Cartan at the Université Henri Poincaré in Nancy, France, for hospitality during his stay in May 2012 where part of the work was done.

\section{REFERENCES}

[1] J.D. Avrin, Large-eigenvalue global existence and regularity results for the Navier-Stokes equation, J. Differential Equations 127 (1996), 365-390.

[2] H. Bellout, S. Benachour, and E. Titi, Finite time regularity versus global regularity for hyperviscous HamiltonJacobi-like equations. Nonlinearity 16 (2003), 1967-1989.

[3] P. Collet, J.-P. Eckmann, H. Epstein, and J. Stubbe, A global attracting set for the Kuramoto-Sivashinsky equation. Comm. Math. Phys. 152 (1993), no. 1, 203-214.

[4] L. Giacomelli and F. Otto, New bounds for the Kuramoto-Sivashinsky equation. Comm. Pure Appl. Math. 43 (2005), 297-318.

[5] J. Goodman, Stability of the Kuramoto-Sivashinsky and related systems. Comm. Pure Appl. Math. 47 (1994), no. 3, 293-306.

[6] L. Hoang and G. R. Sell, Navier-Stokes equations with Navier boundary conditions for an oceanic model. (English summary) J. Dynam. Differential Equations 22 (2010), no. 3, 563-616.

[7] L. Hoang, Incompressible fluids in thin domains with Navier friction boundary conditions (I). J. Math. Fluid Mech. 12 (2010), no. 3, 435-472.

[8] Y.S. Ilyashenko, Global analysis of the phase portrait for the Kuramoto-Sivashinsky equation. J. Dynam. Diff. Eq. 4 (1992), no. 4, 585-615.

[9] I. Kukavica and M. Malcok, Backward behavior of solutions of the Kuramoto-Sivashinsky equation. J. Math. Anal. Appl. 307 (2005), no. 2, 455-464.

[10] I. Kukavica, W. Rusin, and M. Ziane, A class of solutions of the Navier-Stokes equations with large data. preprint.

[11] L. Molinet, Local dissipativity in $L^{2}$ for the Kuramoto-Sivashinsky equation in spatial dimension 2. J. Dynam. Diff. Eq. 12 (2000), no. 3, 533-556.

[12] B. Nicolaenko, B. Scheurer, and R. Temam, Some global dynamical properties of the Kuramoto-Sivashinsky equations: nonlinear stability and attractors. Phys. D 16 (1985), no. 2, 155-183. 
[13] F. Otto, Optimal bounds on the Kuramoto-Sivashinsky equation. J. Funct. Anal. 257 (2009), no. 7, $2188-2245$.

[14] D.T. Papageorgiou and D. Tseluko, A global contracting set for nonlinear KuramotoSivashinsky equations arising in interfacial electrohydrodynamics. European J. Appl. Math. 17 (2006), 677-703.

[15] G. Raugel and G. R. Sell, Navier-Stokes equations on thin 3D domains. I. Global attractors and global regularity of solutions. J. Amer. Math. Soc. 6 (1993), no. 3, 503-568.

[16] G. Raugel and G. R. Sell, Navier-Stokes equations on thin 3D domains. II. Global regularity of spatially periodic solutions, in "Nonlinear Partial Differential Equations and Their Applications," Collège de France Seminar, Vol. XI, pp. 205-247, Longman, Harlow, 1994.

[17] G. Raugel and G. R. Sell, Navier-Stokes equations in thin 3D domains. III. Existence of a global attractor. Turbulence in fluid flows, 137163, IMA Vol. Math. Appl., 55, Springer, New York, 1993.

[18] M. Rost and J. Krug, Anisotropic Kuramoto-Sivashinsky equation for surface growth erosion. Phy. Rev. Lett. bf 75 (1995), no. 21, 3894-3897.

[19] G.R. Sell and M. Taboada, Local dissipativity and attractors for the Kuramoto-Sivashinsky equation in thin $2 D$ domains. Nonlin. Anal. 18 (1992), 671-687.

[20] G. Sivashinsky, On the flame propagation under conditions of stochiometry. SIAM J. Appl. Math. 75 (1980), $67-82$.

[21] R. Temam, Infinite-Dimensional Dynamical Systems in Mechanics and Physics Springer, Berlin-HeidelbergNew York (1988).

[22] R. Temam and M. Ziane, Navier-Stokes equations in three-dimensional thin domains with various boundary conditions, Adv. Differential Equations 1 (1996), 499-546.

Université de Lorraine, Institut Elie Cartan, F-54506 Vandeuvre- lès-Nancy Cedex, France.

E-mail address: said.benachour@univ-lorraine.fr

Department of Mathematics, University of Southern California, 3620 S. Vermont Ave., KaP 108, Los ANGeles, CA 90089

E-mail address: kukavica@usc.edu

Department of Mathematics, University of Southern California, 3620 S. Vermont Ave., Kap 108, Los Angeles, CA 90089

E-mail address: wrusin@usc.edu

Department of Mathematics, University of Southern California, 3620 S. Vermont Ave., Kap 108, Los Angeles, CA 90089

E-mail address: ziane@usc.edu 